\title{
iTRAQ-Based Differential Proteomic Analysis Reveals the Pathways Associated with Tigecycline Resistance in Acinetobacter baumannii
}

\author{
Ni Yang Yuan Liu Ping He Rui Ke Yujie Zhao Yanjing Feng \\ Rong Jing Shuzhen Ma Congrui Liu Yan Geng Xiaokang Wu \\ Yang Wei
}

The Second Affiliated Hospital of Xi'an Jiaotong University, Xibei Hospital, Xi'an, China

\section{Key Words}

Acinetobacter baumannii $\bullet$ iTRAQ $•$ Drug resistance $\cdot$ Tigecycline $\bullet$ Protein degradation

\begin{abstract}
Background/Aims: Acinetobacter baumannii is an aerobic and Gram-negative bacterial pathogen with high morbidity and mortality. It remains a serious public health problem arising from its multidrug-resistant and extensive antibiotic resistance spectrum. Methods: In the present study, iTRAQ coupled with 2D LC-MS/MS was used to evaluate the proteome in standard Acinetobacter baumannii standard strains and tigecycline-resistant strains. Results: A total of 3639 proteins were identified and 961 proteins were identified to be differentially expressed in tigecycline-resistant Acinetobacter baumannii strains compared to the standard strains. 506 (52.6\%) proteins were up-regulated and 455 (47.4\%) proteins were down-regulated. Based on the GO enrichment analysis and KEGG pathway analysis, we concluded that most differentially expressed proteins were associated with stress responses, cellular component organization, proteins synthesis, degradation and function. Moreover, $\beta$-lactam resistance, the longevity regulating pathway and other related pathways were also involved in the regulation of tigecycline-resistant Acinetobacter baumannii. The differential expression of key proteins were evaluated by transcript analysis using quantitative RT-PCR. Conclusion: These results may provide new insights into the mechanisms of drug resistance in Acinetobacter baumannii.




\section{Cellular Physiology Cell Physiol Biochem 2018;51:1327-1339

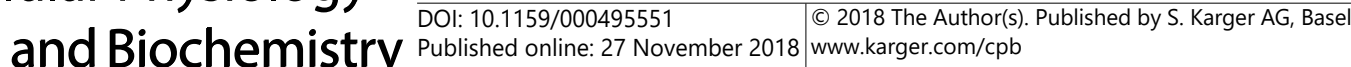 \\ Yang et al.: Proteomic Profiling of Acinetobacter Baumannii}

\section{Introduction}

Acinetobacter baumannii is an aerobic and Gram-negative bacterial pathogen with high morbidity and mortality. As its pleomorphic and opportunistic character, Acinetobacter baumannii has a higher incidence of nosocomial infections and often leads to the occurrence of various diseases, such as bacteremia, urinary tract infections, meningitis, pneumonia and others $[1,2]$. More remarkable, it has been recognized as a severe human pathogen and a intractable challenge to the medical fraternity, arising from its multidrug-resistant and extensive antibiotic resistance spectrum, including tetracyclines, fluoroquinolones, $\beta$-lactams and colistin $[3,4]$.

Known risk-factors for the development of drug-resistant Acinetobacter baumannii are diverse, especially the overuse of antibiotics [5]. In these antibiotics, tigecycline, a member of glycylcyclines, is often one of the available antibiotics remaining treatment to Acinetobacter baumannii, although tigecycline-resistant clinical Acinetobacter baumannii isolates have increasingly been reported [6]. In terms of Acinetobacter baumannii, there have been numerous researches about the mechanism involved in the progress of drug resistance, however, the significant improvement in the therapeutic strategies is still very poor [7]. Hence, furthermore knowledge about the mechanism of drug resistance in Acinetobacter baumannii are urgently needed to improve the therapeutic options of infection.

Proteomic analysis based on mass spectrometry and bioinformatics is powerful technique for the high-throughput characterization of global differential expressed protein [8]. The proteomics techniques have been applied in previous studies to characterize the potential features of multidrug-resistant Acinetobacter baumannii from different perspectives $[9,10]$. Whereas, the comparison of expression difference in global proteins between Acinetobacter baumannii standard strains and tigecycline-resistant strains have not been reported.

In the present study, we employed the isobaric tag for relative and absolute quantitation (iTRAQ) coupled with 2D nano LC-MS/MS, a powerful quantitative tool in proteomics studies, to perform the analysis of differential global proteins in these two activated state of Acinetobacter baumannii strains. These results might provide a novel understanding of the drug resistance in Acinetobacter baumannii.

\section{Materials and Methods}

\section{Sample preparation}

Using multi-step inducement to acquire Tigecycline resistant strain (Fig. 1). Activated the Acinetobacter baumannii ATCC19606 strain on blood agar, and serially passaged for five times. The MIC Test Strip (MTS) method was used to determine tigecycline susceptibility of the original ATCC19606 and the quality control strain ATCC25922, and the MIC were $0.75 \mathrm{ug} / \mathrm{ml}, \quad 0.064 \mathrm{ug} / \mathrm{ml}$. Then inoculated the single colony of the ATCC19606 which obtained from the last generation into 0.5 times MIC concentration of tigecycline in LB broth. Meanwhile, the LB broth contained bacteria without tigecycline was set as an negative control. The above culture solution were shaken overnight at $200 \mathrm{rpm} / \mathrm{min}, 37^{\circ} \mathrm{C}$, and changed the medium at the second day. Repeated the above process, and cultured for 3 generations and then measured the MIC of tigecycline using the MTS assay. And then continually inoculated the strains in the one-half MIC values. With the concentration of tigecycline increased, the cultivate generation increased also. Repeated the progress, and the level of resistance was obtained. The MIC of tigecycline of the experimental group, negative control group and quality control group were $16 \mathrm{ug} / \mathrm{ml}, 1 \mathrm{ug} / \mathrm{ml}, \quad 0.064 \mathrm{ug} / \mathrm{ml}$. The stability of the tigecycline MIC for 19606 was tested by 10 passages without selective pressure.

\section{SDS-PAGE analysis}

SDS-PAGE was performed to determine the quality of isolated protein samples (Supplementary Figure S1 For all supplemental material see www.karger.com/10.1159/000495551). Briefly, $1 \mathrm{~g}$ bacteria was collected and grinded with liquid nitrogen, followed by dissolving with $500 \mu$ SDT lysate (4\% SDS, $100 \mathrm{mM}$ tris-hcl,1 mM DTT, pH 7.6). The samples were then kept in boiling water for 5 min, and lysed by ultrasonic crushing. After centrifugation, protein samples were collected and quantified using BCA assay. And $20 \mu \mathrm{g}$ samples were taken for SDS-PAGE. Then the gels were stained with coomassie brilliant blue G250 
and analyzed. The quality of samples is proper for proteomic analysis.

\section{Protein preparation and ITRAQ labeling}

Total proteins were isolated from suitable strains. And the Bradford method was used to determine protein concentrations. Three biological replicates were carried out for activited Acinetobacter baumannii strain and the control strain, respectively. Equal amount of proteins were treated with DTT and iodoacetamide, then digested using sequencing grade trypsin (ratio of $1: 10 \mathrm{w}: \mathrm{w})$ overnight at $37{ }^{\circ} \mathrm{C}$. The resulting peptide mixture was labeled using the 8-plex iTRAQ kits (AB Sciex, MA, USA) according to the manufacturer's instructions. Three samples from the control strains (C) were labeled with iTRAQ tags 113,114 and 115. The other three samples from the activited strain (T) were labeled with iTRAQ tags 116, 117 and 118.

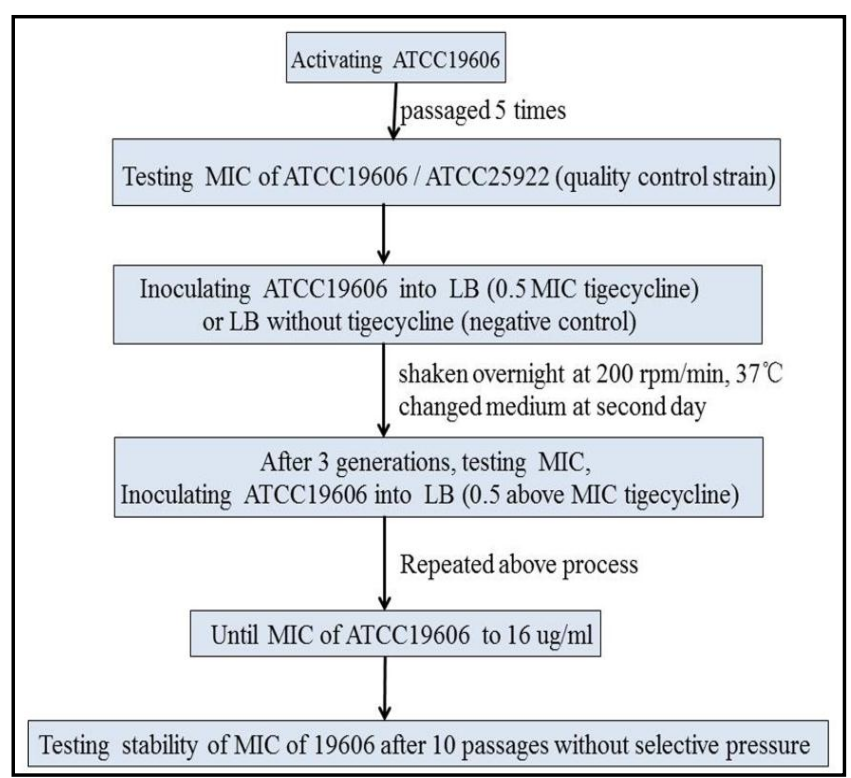

Fig. 1. The work flow figure of inducing Tigecycline resistant Acinetobacter baumannii.

\section{Strong cationic-exchange chromatography separation}

After acidified with $1 \%$ trifluoroacetic acid, the combined peptide mixture were dissolved in strong cation exchange (SCX) buffer A, and fractionated using the Ultremex SCX column (Poly LC, Columbia, MD). The solvents were subjected with a gradient of solvent B, and was monitored by absorbance at $214 \mathrm{~nm}$. The fractions were then collected every $1 \mathrm{~min}$, Finally, 30 collected fractions were pooled into 15 samples, followed by drying and desalting before LC-MS/MS analysis.

\section{LC-MS/MS analysis}

LC-MS/MS analysis was performed as described previously [8]. $5 \mu \mathrm{g}$ of peptides from each fraction were resolved in solvent C, and separated using Shimadzu LC-20AD Nano-HPLC system with a linear gradient of solvent D at a flow rate of $300 \mathrm{~nL} / \mathrm{min}$ over $120 \mathrm{~min}$. Solvent D was added in a linear gradient from $0 \%$ solvent D to $45 \%$ solvent D over $100 \mathrm{~min}$, followed by ramping up to $100 \%$ solvent D over $8 \mathrm{~min}$, up to $100 \%$ solvent D in $12 \mathrm{~min}$. MS/MS data were acquired using the Q-Exactive mass spectrometer in the positive ion mode (mass range of 300-800 mass/charge (m/z)). Q-Exactive survey scans were 70, $000 \mathrm{at} \mathrm{m/z} 200$ and 17,500 at m/z 200 of resolution for HCD spectra. Dynamic exclusion was set as $40.0 \mathrm{~s}$ duration. Data were collected in a data-dependent acquisition method using the top 10 most abundant precursor ions. $0.1 \%$ on the Q-Exactive was defined as the underfill ratio, and the normalized collision energy was $30 \mathrm{eV}$.

\section{Protein identification and quantification}

Protein identifications were performed using the MASCOT search engine (version 2.2.1; Matrix Science, London, UK) based on the protein sequences, searching against the Uniprot database of Acinetobacter baumannii (20151216, 587789 entries, downloaded from: http://www.uniprot.org/). Search parameters were set as follows: monoisotopic mass, trypsin as the enzyme, fragment mass tolerance at $0.1 \mathrm{Da}$, peptide mass tolerance at $\pm 20 \mathrm{ppm}$, allowing up to two missed cleavages. Lysine and N-term of peptides labeled by iTRAQ 8-plex, variable modifications were defined as oxidation of methionine and iTRAQ 8-plex labeled tyrosine. False discovery rate (FDR) of peptides identification as set to be less than 0.01 . For quantitative changes, the Proteome Discoverer 1.3 was applied. The up-regulated and down-regulated proteins was determined as 1.5 -fold cutoff with a p-value $<0.05$ in at least two replicates.

\section{Bioinformatics analysis}

The altered protein profiles were identified in activited Acinetobacter baumannii strain by K-means clustering analysis using the Cluster3.0 software and the Java Treeview software. Blast2GO software was 


\section{Cellular Physiology Cell Physiol Biochem 2018;51:1327-1339

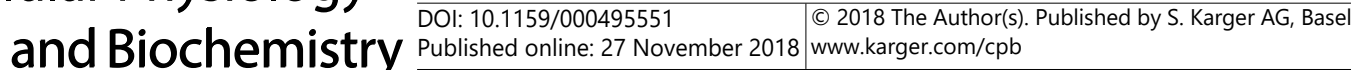 \\ Yang et al.: Proteomic Profiling of Acinetobacter Baumannii}

used to perform the functional annotation analysis. Functional subcategories and metabolic pathways related to the differentially expressed proteins were identified by GO and pathway enrichment analysis. KEGG databases was employed to further reveal the potential biochemical pathways and downstream biological processes.

qRT-PCR

qRT-PCR was performed to evaluate the expression of protein of interest in Acinetobacter baumannii strains. Total RNA was isolated using Trizol (Sangon Biotech, Shanghai, China). PrimeScript@RT Reagent Kit (Takara, Dalian, China) was used to conduct reverse transcription reactions. The primers were designed and synthesized by Sangon Biotech (Supplementary Table S1). Ct values of 16s rRNA was used as the internal control.

\section{Results}

\section{Global profiling of proteins in Acinetobacter baumannii strains}

In order to demonstrate the physiological changes in tigecycline-resistant Acinetobacter baumannii strains, the differences in cellular proteome between Acinetobacter baumannii standard strains and tigecycline-resistant strains were evaluated using an iTRAQ analysis coupled with 2D nano LC-MS/MS (PXD010021 for ProteomeXchange). The quality of samples is proper for proteomic analysis (Supplementary Fig. S2 and S3). According to previous studies, any proteins changed with 1.5 -fold cutoff and a $\mathrm{P}$ value $<0.05$ would be determined as significant differently expression proteins. By untargeted proteomic analysis and quantitative detecting proteins in two activated state of Acinetobacter baumannii strains, a total of 3639 proteins was identified, in which 961 of these proteins were quantified to be differentially expressed in tigecycline-resistant Acinetobacter baumannii strains compared to the standard strains with high confidence (Supplementary Table S2 and S3).

\section{Identification of differentially expressed proteins}

Based on the K-means clustering analysis, we found that there were 506 (52.6\%) proteins up-regulated and 455 (47.4\%) proteins down-regulated in the tigecycline-resistant strains (Supplementary Table S2 and S3), including various outer membrane protein, efflux pump participator, enzymes and others. The visualized heat map displayed the K-means clustering of those differentially regulated proteins in two different strains (Supplementary Fig. S4). Heat map of spots also showed the accumulation patterns of differentially expressed proteins in tigecycline-resistant Acinetobacter baumannii strains. The molecular mass of differentially expressed proteins ranged from $3 \mathrm{kDa}$ to $218 \mathrm{kDa}$, with almost $90 \%$ between $20 \mathrm{kDa}$ and $80 \mathrm{kDa}$. The pI mainly ranged from 4.07 to 11.27 (Supplementary Table S2 and S3).

\section{GO analysis of differentially expressed proteins}

We further conducted the GO annotation analysis to get insight into the functional annotation that was altered in tigecycline-resistant Acinetobacter baumannii strains compared to the standard strains. Based on the GO annotation analysis, differentially expressed proteins were classified into three groups: biological process, molecular function and cellular component. The top $30 \mathrm{GO}$ functional annotation associated with differentially expressed proteins in tigecycline-resistant Acinetobacter baumannii strains were shown in Fig. 2. The most proteins in biological processes categories were in the signaling process, response process to stimulus, cellular protein metabolic process and cellular component organization or biogenesis process. For the molecular functional annotation, the most proteins were mapped to GO terms for protein binding and catalytic activity. In the cellular components category, most proteins were predominantly distributed in the virion, cellular organelle and membrane. 
Additionally, we performed the GO enrichment analysis to further reveal the potential function of differentially expressed proteins in tigecycline-resistant Acinetobacter baumannii strains. The top 30 enriched functional categories associated with differentially expressed proteins in tigecycline-resistant strains were shown in Fig. 3. The majority of proteins were found in the biological processes of intracellular localization, transport, response process to stimulus and amine metabolic process. In the molecular functional category, the most proteins were mapped to cell periphery and acetyltransferase activity. And the most proteins for cellular components were predominantly distributed in the external envelope structure and outer membrane, which might play crucial role in the alteration of membrane permeability in tigecyclineresistant Acinetobacter baumannii.

\section{KEGG pathway analysis} of differentially expressed proteins

In order to reveal the functional categories related to metabolic pathways that responded to tigecycline-resistance in Acinetobacter baumannii strains, KEGG pathway analysis of differentially expressed proteins was then

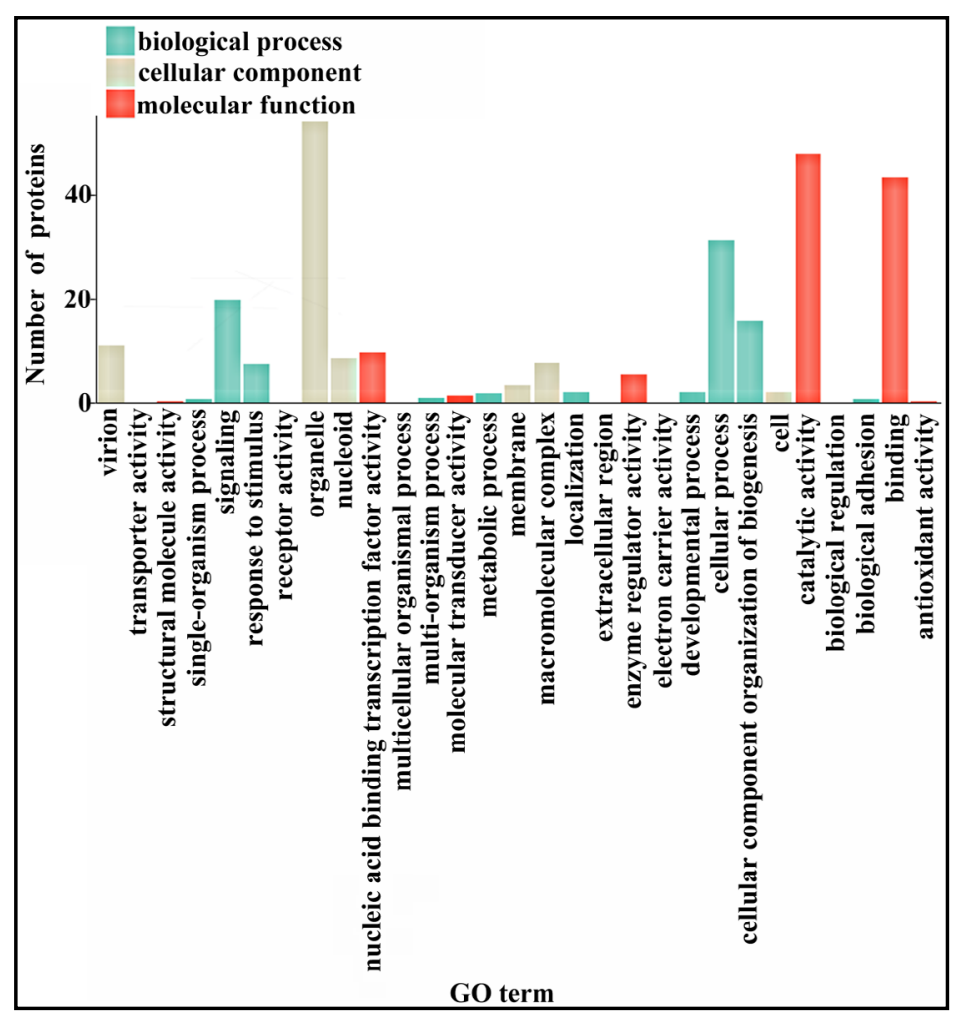

Fig. 2. GO functional annotation of differentially regulated proteins in two different strains.
Fig. $\quad 3 . \quad \mathrm{GO}$ e $\mathrm{n} \mathrm{r}$ c h m e $\mathrm{nt}$ analysis d ifferentially regulated proteins in two different strains.

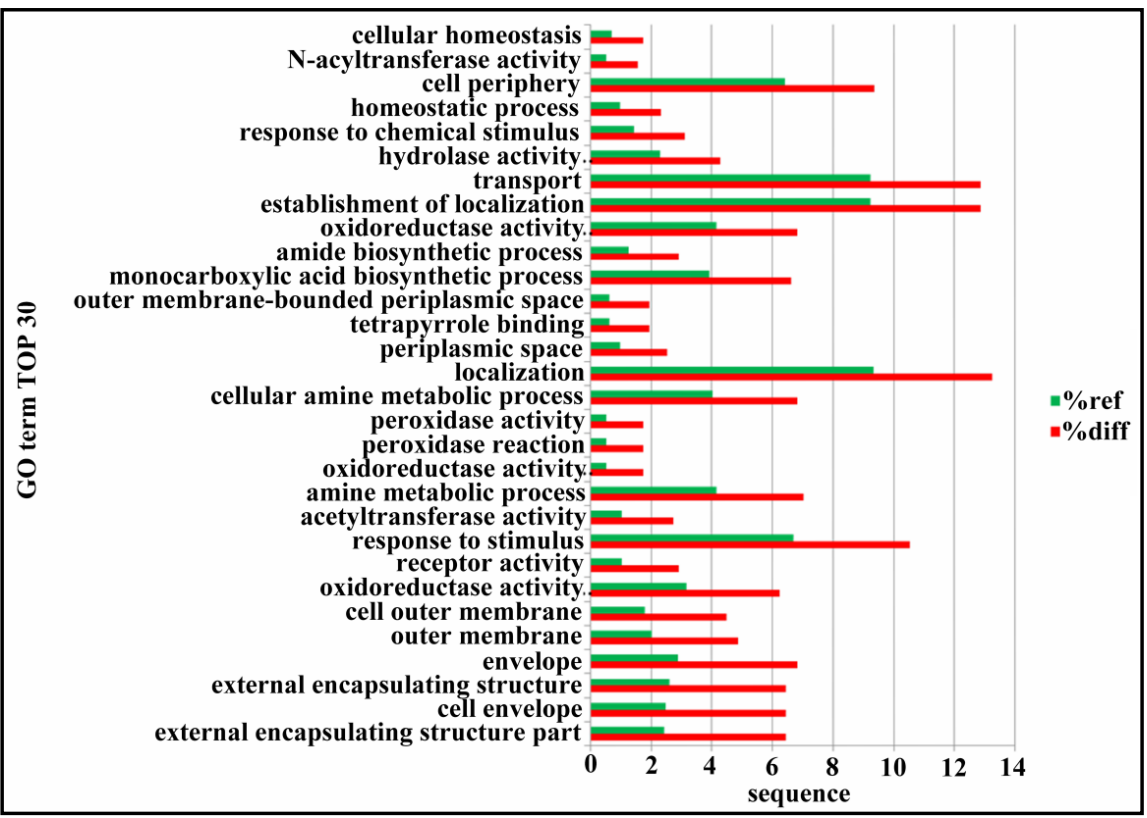




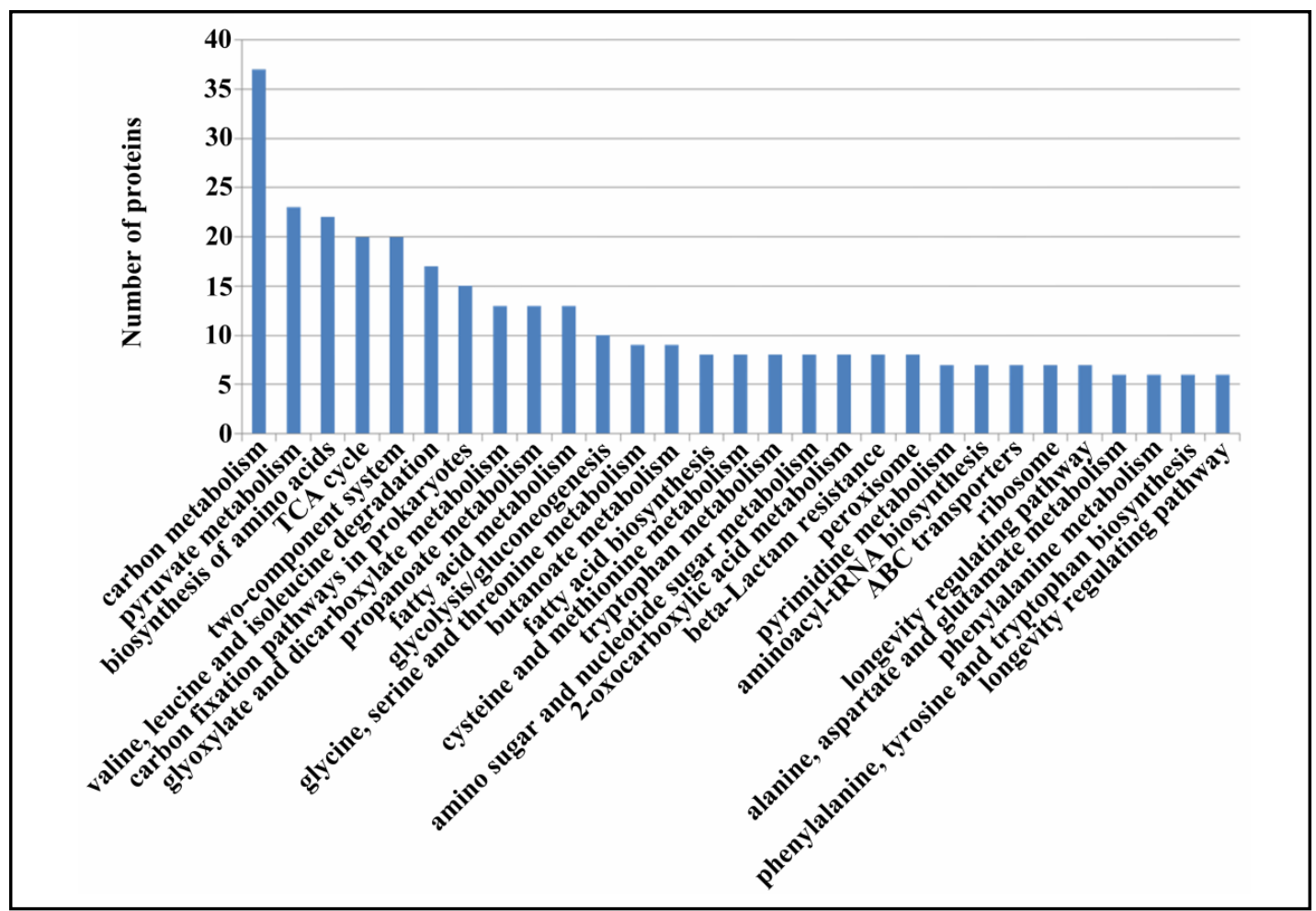

Fig. 4. The most represented KEGG maps.

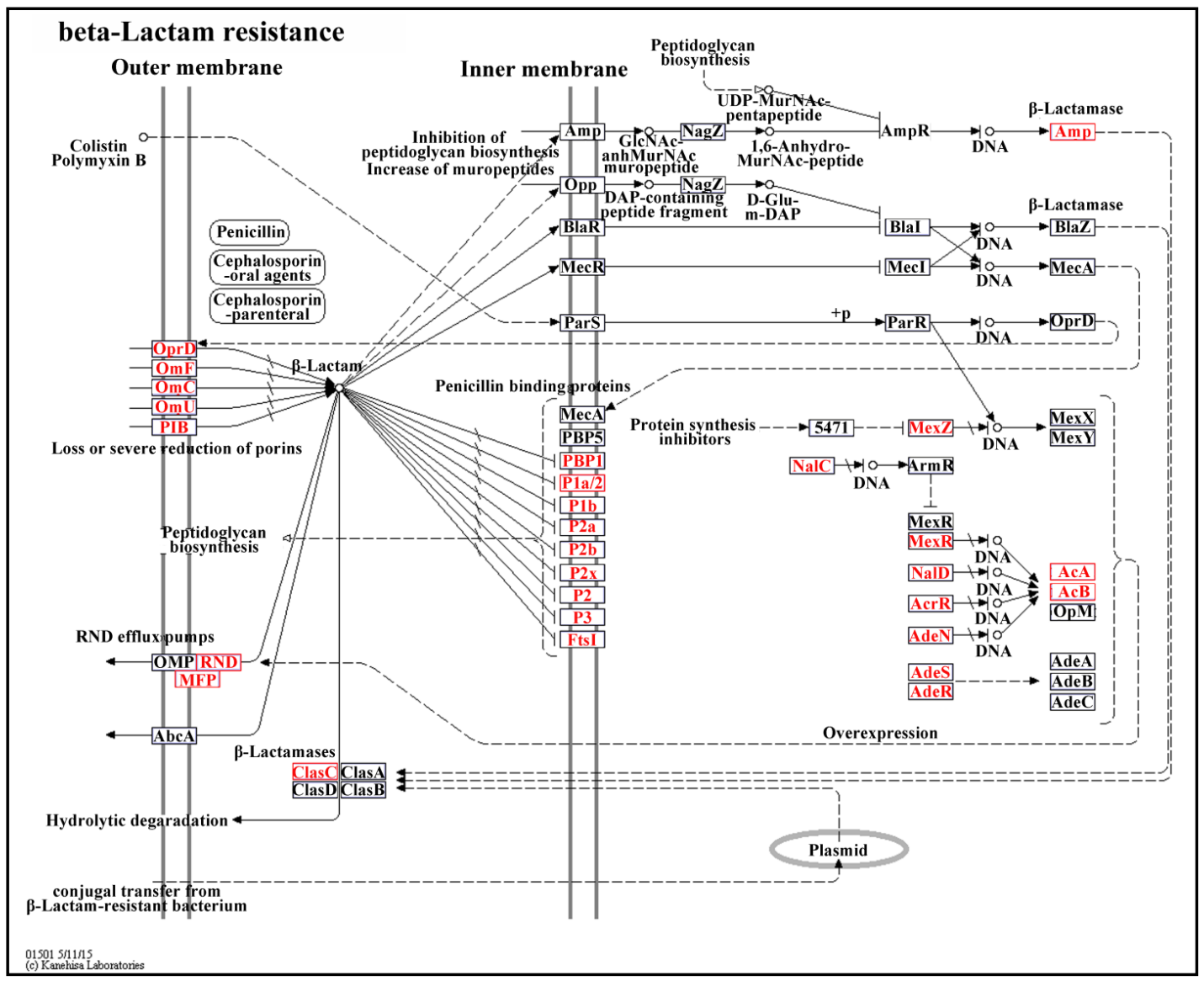

Fig. 5. Representative KEGG pathway for $\beta$-lactam resistance. 

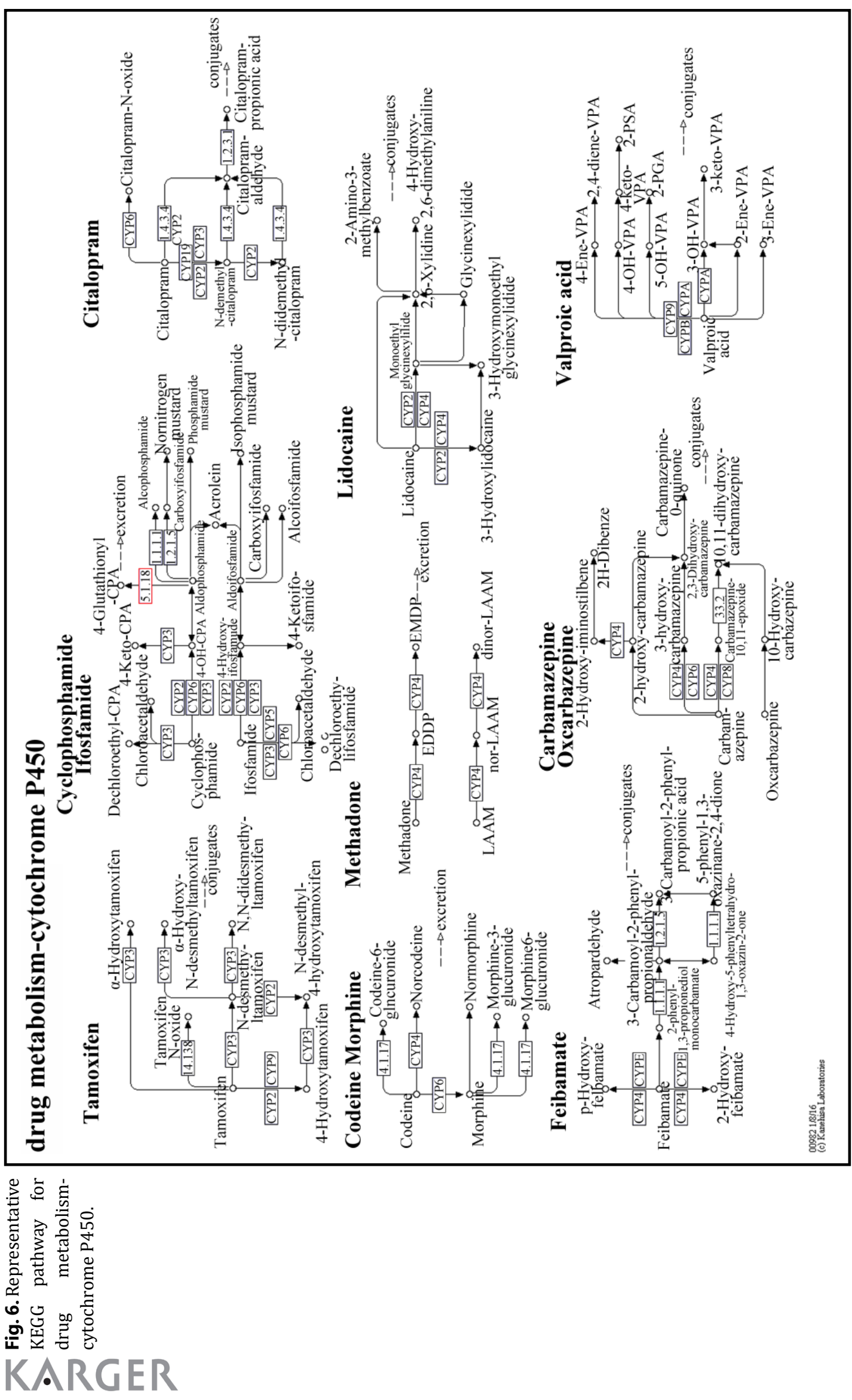

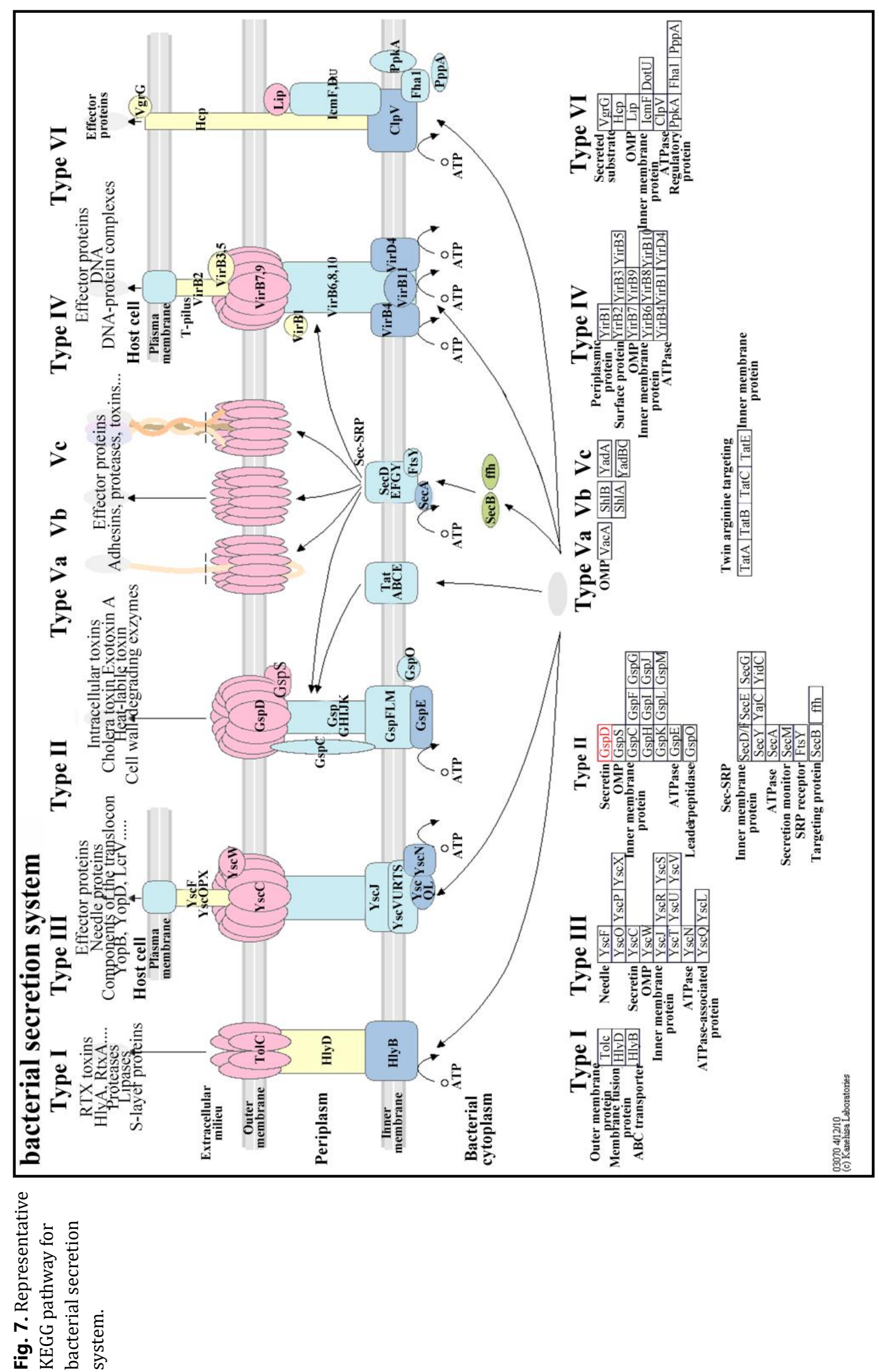


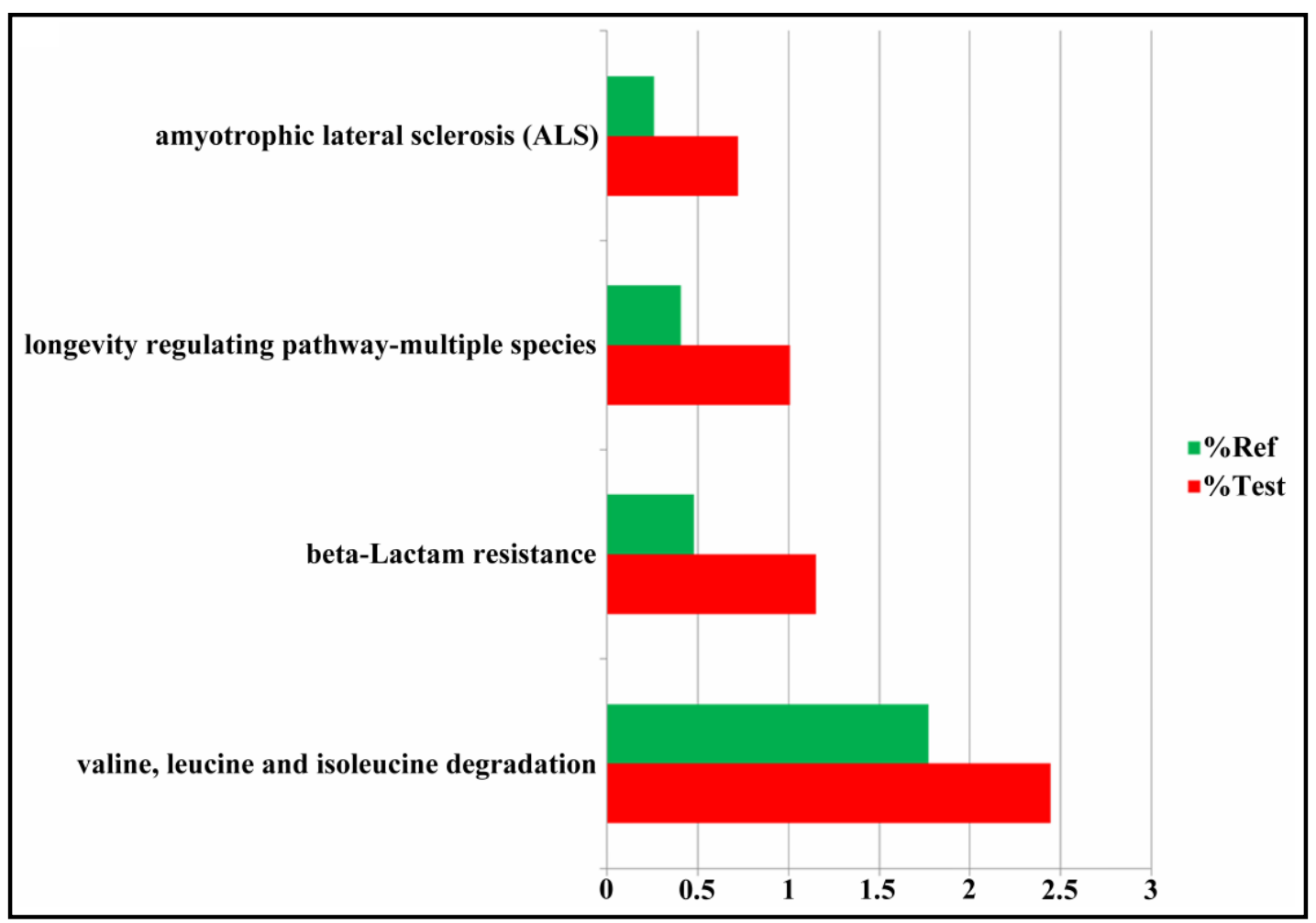

Fig. 8. KEGG pathway analysis of differentially regulated proteins in two different strains.

performed. In this study, we established 138 KEGG pathway associated with all differentially expressed proteins in tigecycline-resistant strains. The most represented KEGG maps were proteins involved in carbon metabolism, amino acids biosynthesis/degradation, $\beta$-lactam resistance, ribosome and others (Fig. 4). Besides, there were also specific KEGG pathways observed that some differentially expressed proteins were involved in, including drug metabolism-cytochrome P450, bacterial secretion system, RNA degradation and others. These pathways might have potential effects on the membrane permeability and activity of efflux pumps in Acinetobacter baumannii strains. The proteins with differential abundances involved in $\beta$-lactam resistance, drug metabolism-cytochrome $\mathrm{P} 450$ and bacterial secretion system were searched in KEGG (http://www.genome.jp/kegg/) and colored in Fig. 5, 6, 7, respectively.

Furthermore, we conducted the KEGG enrichment analysis to further reveal the potential metabolic pathways involved in the tigecycline resistance in Acinetobacter baumannii strains. As shown in Fig. 8, the most proteins were mainly associated with the longevity regulating pathway, $\beta$-lactam resistance and amino acid degradation, which might contribute to the destruction of outer membrane permeability in tigecycline-resistant Acinetobacter baumannii.

\section{Protein-protein interaction analysis}

We then performed a protein-protein interaction analysis by STRING Database version 9.0. As seen in Fig. 9, the differentially expressed proteins were mainly distributed in DNA replication, ribosome, RNA degradation, protein degradation and amino acid metabolism. These processes were all associated with the expression and degradation of proteins, implying the potential changes of protein composition in Acinetobacter baumannii. And this was consistent with the possible alteration of outer membrane and efflux pumps that involved in the tigecycline-resistance. 


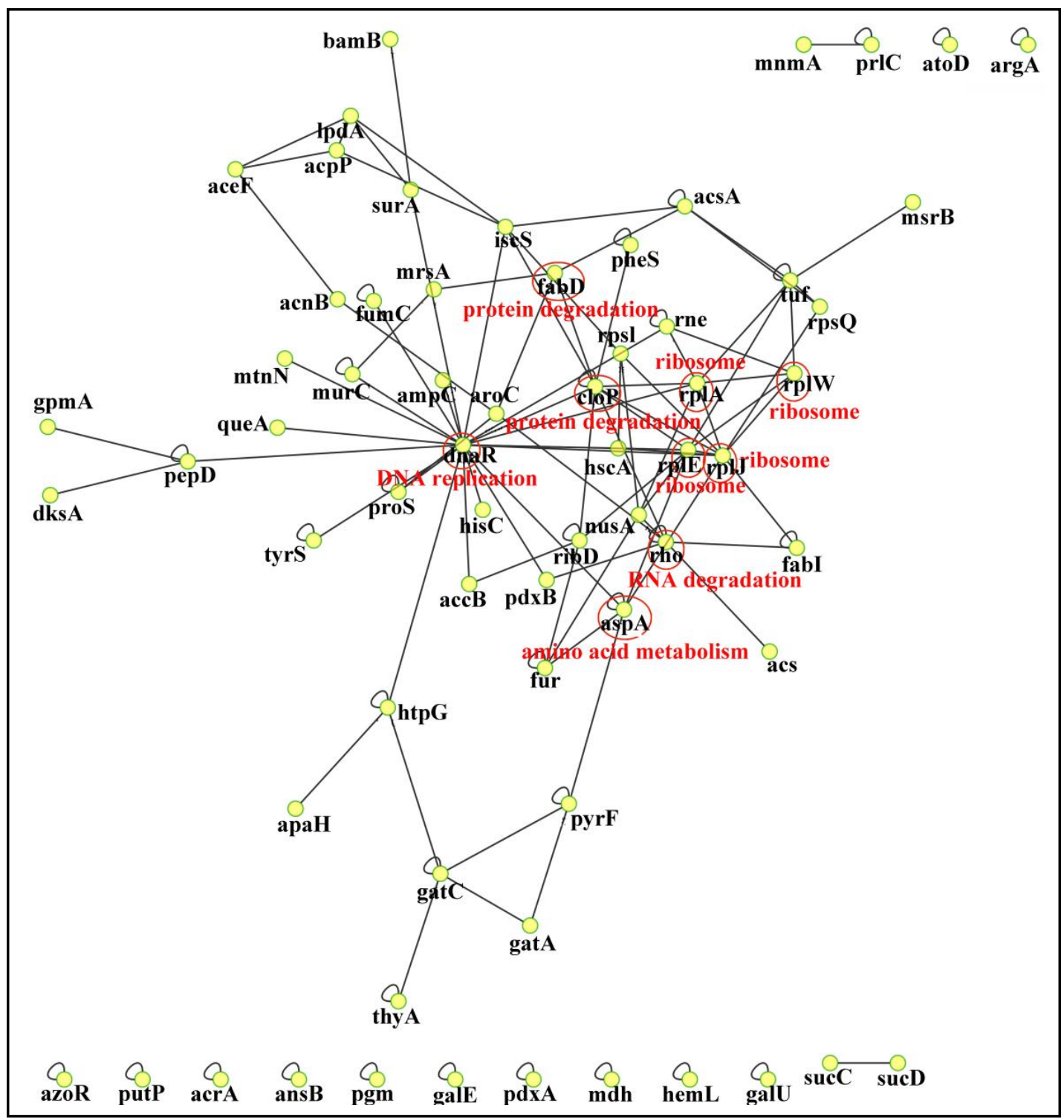

Fig. 9. Protein-protein interaction networks.

Transcript analysis by qRT-PCR

Based on the proteomic analysis, we selected 3 representative proteins for expression analysis at the translational level by qRT-PCR. Results showed that two (A0A009GEE5 and A0A0J8TTR3) of the selected proteins had positive correlations between the expression quantities of transcript and protein levels. And A0A009ERT7 exhibited reverse trend between transcript and protein (Fig. 10 and Supplementary Table S2).

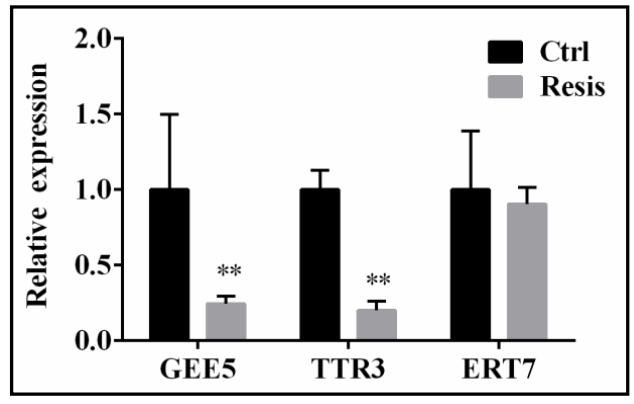

Fig. 10. qRT-PCR analysis of representative proteins in tigecycline-resistant Acinetobacter baumannii strains (Resis) compared to the standard strains (Ctrl) ( ${ }^{* *} \mathrm{P}<0.01$ vs. Ctrl). 


\section{Cellular Physiology Cell Physiol Biochem 2018;51:1327-1339

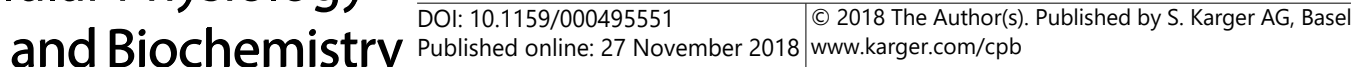 \\ Yang et al.: Proteomic Profiling of Acinetobacter Baumannii}

\section{Discussion}

With different from usual biochemical approaches that only evaluate one or a few of specific proteins, proteomic analysis coupled with 2D LC-MS/MS is a non-targeted research strategy of gene expression, and can monitor the expression of large numbers of genes directly at protein level. This methods can provide integrated insights into the altered expressive status of global proteins under different physiological or pathological conditions [11]. Concomitant analysis of proteomics results from clinical Acinetobacter baumannii isolates has facilitated our understanding of how this bacterial pathogen responds to multidrug. Several transcriptomic and proteomic analyses of antibiotic resistance in Acinetobacter baumannii have been performed $[12,13]$. In the present study, we examined the molecular responses of Acinetobacter baumannii to tigecycline by iTRAQ technology. Our proteomics analysis has provided a novel comparison between the standard Acinetobacter baumannii standard strains and tigecycline-resistant strains.

Nowadays, Acinetobacter baumannii has become a significant clinical concern in nosocomial environments because of its remarkable ability of multidrug-resistance worldwide [2]. To cope with the ability to acquire antibiotic resistance, Acinetobacter baumannii have evolved complex differences in the protein expression, responsive signaling and metabolic processes, especially the key gene expression, at the cellular, organ as well as whole-pathogen levels $[14,15]$. Tigecycline is regarded as one of the few therapeutic options in the control of infections caused by Acinetobacter baumannii. However, tigecyclineresistant clinical Acinetobacter baumannii isolates have increasingly been reported [16].

Antibiotics nonsusceptibility in Acinetobacter baumannii has been identified to be associated with multiply participant factors, including the alterations in outer membrane $(\mathrm{OM})$ permeability, the recruitment of existing enzymes, modifications in the drug-binding proteins and increased expression of efflux pumps [14, 17, 18]. Among them, outer membrane proteins are responsible for the influx of drugs in gram-negative pathogen Acinetobacter baumannii. Compared with the standard Acinetobacter baumannii strains, tigecycline-resistant strains show the alteration in outer membrane permeability $[19,20]$. And this is accompanied by significant changes in the expression of outer membrane proteins in Acinetobacter baumannii strains. The loss of outer membrane proteins may be result in resistance to antibiotics in clinical strains of Acinetobacter baumannii [17]. In this study, we found evidence that a number of proteins associated with outer membrane were differently expressed in tigecycline-resistant Acinetobacter baumannii strains, which indicates that the change in outer membrane permeability is associated with the molecular basis of resistance to tigecycline in Acinetobacter baumannii.

Various proteins associated with the DNA replication, ribosome, RNA degradation, protein degradation and amino acid metabolism were found to be differentially expressed throughout in tigecycline-resistant Acinetobacter baumannii. These proteins have been linked with protein expression and metabolism. In normal strains, proteins biosynthesis and degradation maintain a constant ratio to keep the integrity of cellular structure and function under physiological conditions. Contrary, in tigecycline-resistant strains, the differential expressions of proteins may suggest the alteration in protein metabolism, contributing to the change of outer membrane and efflux pumps that involved in the tigecycline-resistance.

It has been reported that the active efflux systems are, at least in part, involved in the reduced susceptibility in Acinetobacter baumannii that response to antibiotics, including tigecycline [19]. As a class of glycylcyclines, tigecycline is modified by a 9-t-butyl-glycylamido side chain on the minocycline, by which the drug can bind to the ribosomes of bacterial pathogen with high affinity. This characteristic makes it possible that tigecycline retain susceptibility to a broad range of bacteria, including the Gram-positive and Gram-negative, and get out of the major resistance mechanisms of pathogen [21]. However, tigecycline resistance has increasingly emerged [22, 23]. In terms of Acinetobacter baumannii, Deng et al. reported that the major clinically relevant efflux pumps are related with the resistance to tigecycline [24]. Tigecycline is one of the broad substrates of these efflux pumps. 


\section{Cellular Physiology Cell Physiol Biochem 2018:51:1327-1339

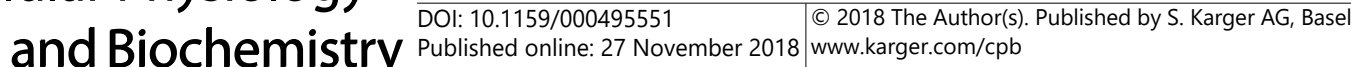 \\ Yang et al.: Proteomic Profiling of Acinetobacter Baumannii}

Through the excretion effect of efflux pumps, tigecycline is reduced to less than effective concentration, resulting in the tigecycline nonsusceptibility in Acinetobacter baumannii. Besides, other possible influence factors of resistance to tigecycline, such as the alterations of existing enzymes or modifications in the drug-binding proteins, were also found in our bio-function analysis, indicating the involvement of these possible mechanisms in resistance to tigecycline in Acinetobacter baumannii. In the present study, the differential expression of two efflux transporter proteins was evaluated by transcript analysis using quantitative RT-PCR, and they had positive correlations between the expression quantities of transcript and protein levels. Notably, an ATP-dependent Clp protease was found to be related with various proteins at PPI networks, and was differential expressed with reverse trend between transcript and protein. We presume that expression of the Clp protease may be regulated by different signaling at transcriptional and protein levels or posttranscriptional modification.

\section{Conclusion}

The proteomic analysis by iTRAQ coupled with 2D LC-MS/MS was conducted to evaluate the proteome in standard Acinetobacter baumannii standard strains and tigecycline-resistant strains. Based on the GO and KEGG pathway analysis, we concluded that most differentially expressed proteins were associated with stress responses, cellular component organization, proteins synthesis, degradation and function. Moreover, $\beta$-lactam resistance, the longevity regulating pathway and other related pathways were also involved in the regulation of tigecycline-resistant Acinetobacter baumannii. These results may provide new insights into the mechanisms of drug resistance in Acinetobacter baumannii.

\section{Acknowledgements}

This work was supported by the National Natural Science Foundation of China (No. H0104) and the Social Development Science and Technology Key Project of Shaanxi Province (No. 2016SF-065). Author Contributions: Ni Yang, Yuan Liu, Ping He, Rui Ke and Yujie Zhao performed all the experiments, data analysis and wrote the paper. Yanjing Feng, Rong Jing and Shuzhen Ma contributed to data analysis. Congrui Liu, Yan Geng, Xiaokang Wu and Yang Wei designed and supervised experiments.

\section{Disclosure Statement}

The authors declare no conflicts of interest regarding the publication of this paper.

\section{References}

1 Longo F, Vuotto C, Donelli G: Biofilm formation in acinetobacter baumannii. New Microbiol 2014;37:119-

127.

-2 Sakellaridis N, Kontoyannis V: Acinetobacter baumannii. J Neurosurg 2013;119:1656.

- Moffatt JH, Harper M, Harrison P, Hale JDF, Vinogradov E, Seemann T, Henry R, Crane B, St. Michael F, Cox AD, Adler B, Nation RL, Li J, Boyce JD: Colistin resistance in acinetobacter baumannii is mediated by complete loss of lipopolysaccharide production. Antimicrob Agents Chemother 2010;54:4971-4977.

- 4 Kuo LC, Lai CC, Liao CH, Hsu CK, Chang YL, Chang CY, Hsueh PR: Multidrug-resistant acinetobacter baumannii bacteraemia: Clinical features, antimicrobial therapy and outcome. Clin Microbiol Infect 2007;13:196-198.

5 Lee HY, Chen CL, Wu SR, Huang CW, Chiu CH: Risk factors and outcome analysis of acinetobacter baumannii complex bacteremia in critical patients. Crit Care Med 2014;42:1081-1088. 


\section{Cellular Physiology Cell Physiol Biochem 2018;51:1327-1339 and Biochemistry \begin{tabular}{l|l|l}
\hline DOI: 10.1159/000495551 2018 The Author(s). Published by S. Karger AG, Basel \\
\hline
\end{tabular}

6 Hua X, Chen Q, Li X, Yu Y: Global transcriptional response of acinetobacter baumannii to a subinhibitory concentration of tigecycline. Int J Antimicrob Agents 2014;44:337-344.

-7 Perez F, Hujer AM, Hujer KM, Decker BK, Rather PN, Bonomo RA: Global challenge of multidrug-resistant acinetobacter baumannii. Antimicrob Agents Chemother 2007;51:3471-3484.

-8 Pan HT, Guo MX, Xiong YM, Ren J, Zhang JY, Gao Q, Ke ZH, Xu GF, Tan YJ, Sheng JZ, Huang HF: Differential proteomic analysis of umbilical artery tissue from preeclampsia patients, using itraq isobaric tags and $2 \mathrm{~d}$ nano lc-ms/ms. J Proteomics 2015;112:262-273.

-9 Méndez JA, Mateos J, Beceiro A, Lopez M, Tomás M, Poza M, Bou G: Quantitative proteomic analysis of host-pathogen interactions: A study of acinetobacter baumannii responses to host airways. BMC genomics 2015;16:422.

10 Moore JL, Becker KW, Nicklay JJ, Boyd KL, Skaar EP, Caprioli RM: Imaging mass spectrometry for assessing temporal proteomics: Analysis of calprotectin in acinetobacter baumannii pulmonary infection. Proteomics 2014;14:820-828.

-11 Jin XJ, Peng HS, Hu HB, Huang XQ, Wang W, Zhang XH: Itraq-based quantitative proteomic analysis reveals potential factors associated with the enhancement of phenazine-1-carboxamide production in pseudomonas chlororaphis p3. Sci Rep 2016;6:27393.

12 Yun SH, Choi CW, Park SH, Lee JC, Leem SH, Choi JS, Kim S, Kim SI: Proteomic analysis of outer membrane proteins from acinetobacter baumannii du202 in tetracycline stress condition. J Microbiol 2008;46:720727.

13 Shin JH, Lee HW, Kim SM, Kim J: Proteomic analysis of acinetobacter baumannii in biofilm and planktonic growth mode. J Microbiol 2009;47:728-735.

14 Shi WF, Jian JP, Mi ZH: Relationship between antimicrobial resistance and aminoglycoside-modifying enzyme gene expressions in acinetobacter baumannii. Chin Med J (Engl). 2005;118:141-145.

15 Ehlers MM, Hughes JM: Antibiotic resistance genes in acinetobacter baumannii isolates; in Kock MM (eds): Antibiotic Resistant Bacteria - A Continuous Challenge in the New Millennium. In Tech, 2012, pp 213-246.

16 Navon-Venezia S, Leavitt A, Carmeli Y: High tigecycline resistance in multidrug-resistant acinetobacter baumannii. J Antimicrob Chemother 2007;59:772-774.

17 Mussi MA, Limansky AS, Viale AM: Acquisition of resistance to carbapenems in multidrug-resistant clinical strains of acinetobacter baumannii: Natural insertional inactivation of a gene encoding a member of a novel family of -barrel outer membrane proteins. Antimicrob Agents Chemother 2005;49:1432-1440.

-18 Fernandez-Cuenca F: Relationship between beta-lactamase production, outer membrane protein and penicillin-binding protein profiles on the activity of carbapenems against clinical isolates of acinetobacter baumannii. J Antimicrob Chemother 2003;51:565-574.

19 Li H: The role of rnd efflux pump and global regulators in tigecycline resistance in clinical acinetobacter baumannii isolates. Future Microbiol 2015;10:337-346.

20 Kwon SH, Ahn HL, Han OY, La HO: Efficacy and safety profile comparison of colistin and tigecycline on the extensively drug resistant acinetobacter baumannii. Biol Pharm Bull 2014;37:340-346.

-21 Ying J, Lu J, Zong L, Li A, Pan R, Cheng C, Li K, Chen L, Ying J, Tou H: Molecular epidemiology and characterization of genotypes of acinetobacter baumannii isolates from regions of south china. Jpn J Infect Dis 2016;69:180-185.

-22 Piewngam P, Kiratisin P: Comparative assessment of antimicrobial susceptibility testing for tigecycline and colistin against acinetobacter baumannii clinical isolates, including multidrug-resistant isolates. Int J Antimicrob Agents 2014;44:396-401.

-23 Baadani AM, Thawadi SI, El-Khizzi NA, Omrani AS: Prevalence of colistin and tigecycline resistance in acinetobacter baumannii clinical isolates from 2 hospitals in riyadh region over a 2-year period. Saudi Med J 2013;34:248-253.

24 Deng M, Zhu MH, Li JJ, Bi S, Sheng ZK, Hu FS, Zhang JJ, Chen W, Xue XW, Sheng JF, Li LJ: Molecular epidemiology and mechanisms of tigecycline resistance in clinical isolates of acinetobacter baumannii from a chinese university hospital. Antimicrob Agents Chemother 2013;58:297-303. 